\title{
LINC01116 promotes the proliferation and invasion of glioma by regulating the microRNA-744-5p-MDM2-p53 axis
}

\author{
LI JIANG $^{1 *}$, CHAO CHENG $^{2 *}$, WEI JI $^{2^{*}}$, HAO WANG $^{1}$, QUAN DU $^{1}$, \\ XIAOQIAO DONG ${ }^{1}$, JUNFEI SHAO ${ }^{2}$ and WENHUA YU ${ }^{1}$ \\ ${ }^{1}$ Department of Neurosurgery, Hangzhou First People's Hospital of Nanjing Medical University, Hangzhou, Zhejiang 310006; \\ ${ }^{2}$ Department of Neurosurgery, Wuxi People's Hospital of Nanjing Medical University, Wuxi, Jiangsu 214023, P.R. China
}

Received January 12, 2020; Accepted January 15, 2021

DOI: $10.3892 / \mathrm{mmr} .2021 .12005$

\begin{abstract}
Long non-coding RNAs (lncRNAs) have been implicated in the development and progression of tumors. However, the roles and underlying mechanisms of long intergenic non-protein coding RNA 1116 (LINC01116), a member of the lncRNA family, in glioma progression are largely unclear. The expression of LINC01116 and microRNA (miR)-744-5p in glioma tissues and cells was detected by reverse transcription-quantitative PCR. The influences of LINC01116 or miR-744-5p on cell proliferation and invasion were evaluated by Cell Counting Kit-8, colony formation and Transwell assays, and western blotting was used to detect the expression of p53 pathway proteins. A dual-luciferase reporter system was used to locate common binding sites between miR-744-5p and LINC01116 or the 3' untranslated region of E3 ubiquitin-protein ligase Mdm2 (MDM2). RNA immunoprecipitation was used to determine the interactions between RNAs and proteins. Moreover, a xenograft mouse model was constructed to investigate the effects of LINC01116 in vivo, followed by a TdT-mediated dUTP nick end labeling assay to determine the degree of apoptosis in nude mouse tumors. LINC01116 was found to be highly expressed in glioma tissues, which was associated with a malignant phenotype. LINC01116 promoted the proliferation and invasiveness of glioma cells,
\end{abstract}

Correspondence to: Professor Wenhua Yu, Department of Neurosurgery, Hangzhou First People's Hospital of Nanjing Medical University, 261 Huansha Road, Hangzhou, Zhejiang 310006, P.R. China

E-mail: neuroywh@163.com

Professor Junfei Shao, Department of Neurosurgery, Wuxi People's Hospital of Nanjing Medical University, 299 Qingyang Road, Wuxi, Jiangsu 214023, P.R. China

E-mail: wxhneurosurgery@126.com

${ }^{*}$ Contributed equally

Key words: glioma, long intergenic non-protein coding RNA 1116, microRNA-744-5p, MDM2, p53 pathway, competing endogenous RNA and inhibited the p53 pathway by preserving the expression of MDM2 mRNA via miR-744-5p sponging. Furthermore, a low degree of miR-744-5p expression was observed in glioma tissues, which was negatively associated with the expression of LINC01116. Overexpression of miR-744-5p inhibited the proliferation and invasiveness of glioma cells, which was rescued by LINC01116. Finally, LINC01116 knockdown inhibited tumor growth in nude mice. In conclusion, LINC01116 is aberrantly expressed and promotes the progression of glioma by regulating the miR-744-5p-MDM2-p53 pathway. In future, targeting LINC01116 may therefore be a potential therapeutic approach for patients with glioma.

\section{Introduction}

Glioma is the most common type of malignant primary brain cancer (1), and is characterized by rapid proliferation, high invasiveness, rapid recurrence and chemo- and radiotherapeutic resistance (2). In spite of the advances in microsurgery and combined treatments, the prognosis of patients with glioma remains unsatisfactory (3). This has driven research to investigate the potential mechanisms underlying the tumorigenesis and development of glioma in order to identify novel therapeutic targets.

A number of studies have demonstrated that the non-coding RNAs [microRNAs (miRNAs/miRs), pseudogenes, long non-coding RNAs (IncRNAs) and circular RNAs] play important roles not only in normal biological processes, but also in the development of tumors $(4,5)$. Complex regulatory networks exist between non-coding RNAs, mRNAs and proteins. A commonly accepted theory is the competing endogenous RNA (ceRNA) hypothesis, through which endogenous RNAs containing certain miRNA binding sites competitively bind the same miRNAs to reduce the suppression of targeted mRNAs, thus retaining the expression of target genes (6). Increasing evidence suggests that the ceRNA mechanism is also involved in tumorigenesis (7-9).

lncRNAs, a group of non-coding RNAs >200 nucleotides in length, are involved in various disease processes, including tumorigenesis $(10,11)$. An increasing number of studies have indicated that the aberrant expression of specific lncRNAs is associated with the development of glioma (12). IncRNAs exert their functions through various mechanisms in an extensive 
array of biological processes, such as the maintenance of stemness, regulation of cell proliferation, tumor angiogenesis and drug resistance (13). In glioma, the functions of lncRNAs include regulating genome activity, posttranscriptional protein modification and location, as well as encoding functional micro-peptides and acting as intercellular communicators (14). However, the mechanisms by which lncRNAs regulate the biological functions of glioma remain to be elucidated. The present study aimed to demonstrate the role of LINC01116 in glioma and its underlying mechanism. The expression levels of LINC01116 in glioma tissue and cell lines were determined by reverse transcription-quantitative (RT-q)PCR. The effect of LINC01116 on cell proliferation, migration and invasion was assessed by cell viability, Transwell and colony formation assays and tumor xenografts in nude mice. In order to identify the underlying mechanism by which LINC01116 exerts its role, bioinformatics analysis and dual-luciferase reporter assay were performed.

\section{Materials and methods}

Cell culture and glioma samples. Glioma (U87MG, A172 and Shg44) and 293T cell lines were purchased from the Cell Resource Center of Shanghai Institutes for Biological Sciences. The U87MG cell line was a version from American Type Culture Collection (ATCC; RRID:CVCL_0022), the origin of which is unknown. The cell line was authenticated by STR profiling, with a $96.55 \%$ match to the ATCC database profile. The HEB normal human astrocyte cell line was purchased from Cobioer; Nanjing Kebai Biotechnology Co., Ltd. All cells were cultured in DMEM (Gibco; Thermo Fisher Scientific, Inc.) with $10 \%$ fetal bovine serum (Gibco; Thermo Fisher Scientific, Inc.) and $1 \%$ penicillin/streptomycin (Beijing Solarbio Science \& Technology Co., Ltd.) at $37^{\circ} \mathrm{C}\left(5 \% \mathrm{CO}_{2}\right.$ and $95 \%$ air $) . \mathrm{A}$ total of 46 fresh glioma samples and 4 normal brain tissue samples (excised from patients with intracerebral hemorrhage) were collected from the Hangzhou First People's Hospital (all specimens were pathologically diagnosed as glioma between January 2010 and October 2019), and stored in liquid nitrogen. The patients of the present study consisted of 27 male patients and 19 female patients. The age range of patients was between 28 and 74 years. Informed written consent was obtained from the patients, agreeing to use their samples for scientific research. All protocols associated with animals or human tissues were approved by the Ethics Committee of Hangzhou First People's Hospital [Hangzhou, China; approval no. 2020(106)-01].

Online cancer database analysis. The expression of LINC01116 in different grades of glioma, as well as their association with overall survival time and the expression of $\mathrm{p} 53$ target genes [BAK1, BAX, cyclin-dependent kinase inhibitor 1 (CDKN1A) and growth arrest and DNA damage-inducible protein GADD45 $\alpha$ (GADD45A)] were analyzed using the Gene Expression Profiling Interactive Analysis (GEPIA) platform (http://gepia.cancer-pku.cn/detail.php) (15).

Matrigel invasion assay. Invasion assays were performed using Transwell plates (diameter, $6.5 \mathrm{~mm}$; aperture, $8.0 \mu \mathrm{m}$; Corning, Inc.) according to the manufacturer's protocols. Briefly, the filters of the upper chamber were precoated with Matrigel
(Becton, Dickinson and Company) at $37^{\circ} \mathrm{C}$ for $30 \mathrm{~min}$, and $1 \times 10^{4}$ glioma cells resuspended in $200 \mu \mathrm{l}$ serum-free medium were added into the chambers. A total of $650 \mu \mathrm{l}$ culture medium was added to the lower compartments with $10 \%$ FBS as a chemoattractant. Following culture at $37^{\circ} \mathrm{C}$ for $24-48 \mathrm{~h}$, the cells in the upper chambers were removed, and cells that had migrated to the lower membrane were fixed with $4 \%$ paraformaldehyde (Beijing Solarbio Science \& Technology Co., Ltd.) for $20 \mathrm{~min}$ and stained with $0.5 \%$ crystal violet solution for $30 \mathrm{~min}$ at room temperature. The images were scanned by Invitrogen EVOS FL AUTO (Thermo Fisher Scientific, Inc.).

Cell proliferation and colony formation assays. A Cell Counting Kit-8 (CCK-8; cat. no. HY-K0301; MedChem Express) assay was used to assess cell viability according to the manufacturer's protocols. Briefly, the indicated cells were seeded into a 96-well plate at a density of $70-80 \%$ cells per well. The culture medium was replaced by serum-free medium and $10 \mu \mathrm{l} \mathrm{CCK}-8$ solution was added at the indicated times. After incubation for $1 \mathrm{~h}$, the OD values were determined at $450 \mathrm{~nm}$. For the colony formation assay, $1 \times 10^{3}$ cells per well were seeded into 6 -well plates (Corning, Inc.). After 14 days of culture, the colonies (>10 cells) were fixed with $4 \%$ paraformaldehyde for $20 \mathrm{~min}$ and stained with $0.5 \%$ crystal violet solution for $30 \mathrm{~min}$ at room temperature. The images were scanned by Scan Wizard EZ (Microtek) and the number of clones was counted using Image J software (National Institutes of Health).

RNA extraction and RT- $q P C R$. Briefly, total RNA was extracted from tissues and the indicated cells using TRIzol ${ }^{\circledR}$ reagent (cat. no. 15596026; Invitrogen; Thermo Fisher Scientific, Inc.), according to the manufacturer's protocols. The RNA was reverse transcribed into cDNA using the PrimeScript ${ }^{\mathrm{TM}}$ RT reagent kit (cat. no. RR047A; Takara Bio, Inc.), and qPCR analyses were conducted according to the manufacturer's protocol using a TB Green ${ }^{\circledR}$ Premix Ex Taq ${ }^{\mathrm{TM}}$ II (cat. no. RR820A; Takara Bio, Inc.) with GAPDH and U6 as the internal controls. The relative expression levels were calculated via the $2^{-\Delta \Delta c q}$ method (16). The sequences of the qPCR primers were as follows: LINC01116 forward, 5'-GTTCAAGTGCGT CCGGGTTT-3' and reverse, 5'-CGGACTTCTTTTCCAGGC GG-3'; miR-744-5p forward, 5'-AATGCGGGGCTAGGG CTA-3' and reverse, 5'-GTGCAGGGTCCGAGGT-3'; BAK1 forward, 5'-GTCAGAAAGTAGTGTCGCCA-3' and reverse, 5'-ACTTGTAGCGTCAGGACAGC-3'; GADD45A forward, 5'-CTGGGAATTTGGCGACGTAA-3' and reverse, 5'-ATG GATGTAGTCTGGGTGCAG-3'; TP53 forward, 5'-CCAAAT ACTCCACACGCAAAT-3' and reverse, 5'-CCTTCCCAG AAAACCTACCAG-3'; MDM2 forward, 5'-GGCTCTGTG TGTAATAAGGGAGA3' and reverse, 5'-GGACTGCCAGGA CTAGACTTTG-3', GAPDH forward, 5'-AGGAGCGAGATC CCGCCAACA-3' and reverse, 5'-CGGCCGTCACGCCAC ATCTT-3'; and U6 forward, 5'-CTCGCTTCGGCAGCACA-3' and reverse, 5'-AACGCTTCACGAATTTGCGT-3'.

Vector construction and transduction. Small hairpin RNA (shRNA) against human LINC01116 (01116 KD; 5'-CCAAAG GCCCTGAAGTACACAGTTT-3') and corresponding negative control (NC) sequences were purchased from Shanghai GeneChem Co., Ltd., and the sequence was inserted into 
a G248 lentiviral vector (Shanghai GeneChem Co., Ltd.). For LINC01116 overexpression (OE) experiments, the indicated cells $\left(1 \times 10^{4}\right)$ were infected with lentivirus containing LINC01116-GV358 plasmids (MOI, 10) at $37^{\circ} \mathrm{C}$ for $12 \mathrm{~h}$, which were synthesized by Shanghai GeneChem Co., Ltd. All transfections were conducted following the manufacturer's protocols. MDM2 small interfering (si)RNA (MDM2 KD) (cat. no. \#AM16708) was acquired from Thermo Fisher Scientific, Inc., and the miR-744-5p mimics (5'-UGCGGG GCUAGGGCUAACAGCA-3') and inhibitor (5'-UGCUGU UAGCCCUAGCCCCCA-3') were designed and synthesized by Shanghai GenePharma Co., Ltd. All transfections were performed using Lipofectamine ${ }^{\circledR} 3000$ (Invitrogen; Thermo Fisher Scientific, Inc.), according to the manufacturer's protocols. The amount of mimics/inhibitor was $2 \mu \mathrm{g} / 2 \times 10^{6}$ cells.

Western blotting. Briefly, total protein was extracted from cells or tissues by incubation with RIPA lysis buffer (Beyotime Institute of Biotechnology) and the protein concentration was measured using a BCA Protein Assay kit (Thermo Fisher Scientific, Inc.). Equal amounts of protein sample ( $20 \mu \mathrm{g} / \mathrm{lane})$ were separated via $10 \%$ SDS-PAGE, and subsequently transferred onto PVDF membranes (Roche Diagnostics). After blocking with skimmed milk (5\%) for $1 \mathrm{~h}$ at room temperature, the membranes were incubated with the following primary antibodies overnight at $4^{\circ} \mathrm{C}$ and secondary antibodies for $1 \mathrm{~h}$ at RT: Anti-p53 (1:1,000; cat. no. 2527), anti-MDM2 (1:1,000; cat. no. 86934), anti-BAK1 (1:1,000; cat. no. 12105), anti-GADD45A (1:1,000; cat. no. 4632), anti- $\beta$-actin (1:1,000; cat. no. 3700), anti-mouse $\operatorname{IgG}(1: 5,000$; cat. no. 7076) and anti-rabbit IgG (1:5,000; cat. no. 7074) from Cell Signaling Technology, Inc. After washing with TBS-Tween-20 (0.05\%), bound HRP-conjugated antibodies were detected using Western Lightning Plus-ECL reagents (PerkinElmer, Inc.). The protein bands were measured by FluorChem Q (ProteinSimple).

Online cancer database analysis. StarBase (starbase.sysu.edu. $\mathrm{cn} /$ ) (17) was used to identify the ceRNA network between LINC01116, microRNA-744-5p and MDM2.

Dual-luciferase reporter assay. Fragments of LINC01116 and the MDM2 mRNA 3' untranslated region (UTR) containing miR-744-5p binding sites, and mutated variants of these fragments, were amplified and subcloned into the pGL3 promoter vector (Promega Corporation). The mutated variants were generated using QuickMutation ${ }^{\mathrm{TM}}$ Site-Directed Mutagenesis kit (cat. no. D0206; Beyotime Institute of Biotechnology), according to the manufacturer's protocol. The indicated plasmids $(1 \mu \mathrm{g})$ and miRNA mimics or inhibitors $(1 \mu \mathrm{g})$ were transfected into 293T cells $\left(2 \times 10^{6}\right)$ using Lipofectamine 3000. After $48 \mathrm{~h}$, luciferase activity was measured using a Dual-Luciferase Reporter Assay Kit (Promega Corporation) according to the manufacturer's protocols. Renilla luciferase was used as the control.

RNA immunoprecipitation (RIP) assay. RIP assays were conducted using the Magna RIP ${ }^{\mathrm{TM}}$ RNA-Binding Protein Immunoprecipitation kit (EMD Millipore), following the manufacturer's protocols. The indicated cells $\left(2 \times 10^{7}\right)$ were incubated with $500 \mu 1$ RIP lysis buffer (Beyotime Institute of Biotechnology). Extracts were incubated with $5 \mu \mathrm{g}$ antibodies against Ago2 (cat. no. 2897; Cell Signaling Technology, Inc.) at $4^{\circ} \mathrm{C}$ overnight, followed by incubating with $30 \mu 1$ Magnetic beads (MedChemExpress; cat. no. HY-K0205) for 6-8 h at $4^{\circ} \mathrm{C}$. Argonaute proteins are involved in the various steps of miRNA-mediated gene silencing by facilitating the formation of micro-ribonucleoproteins complexes with miRNAs $(18,19)$. Rabbit IgG (cat. no. ab172730; Abcam) was used as a negative control. Completes were washed with washing buffer and incubated with proteinase $\mathrm{K}$ at $55^{\circ} \mathrm{C}$ for 30 min to isolate the RNA- protein complexes from beads. RNA was isolated using TRIzol and then reverse-transcribed into cDNA by PrimeScript ${ }^{\mathrm{TM}} \mathrm{RT}$ reagent kit (Takara Biotechnology Co., Ltd.) The levels of precipitated RNA were then determined by RT-qPCR as aforementioned.

Tumor xenografts in nude mice. A total of 30 six-week-old female BALB/c nude mice (weight, 14-20 g) were purchased from The Shanghai Laboratory Animal Center of the Chinese Academy of Sciences. All mice were kept in a temperature-controlled pathogen-free environment $\left(21^{\circ} \mathrm{C}\right)$ on a 12 -h light-dark cycle in accordance with the Guide for the Care and Use of Laboratory Animals. A total of 10 nude mice were randomly divided into two groups for tumor xenografts. The indicated Shg44 cells (5x10 $/ 100 \mu \mathrm{l}$ in primary DMEM) were subcutaneously injected into the flank of each nude mouse, and tumor volume was recorded every 6 days. The tumor volumes were calculated using the following formula: Tumor volume $\left(\mathrm{mm}^{3}\right)=\left(\right.$ length $\mathrm{x}$ width $\left.{ }^{2}\right) / 2$. After 3 weeks, the mice were sacrificed by cervical dislocation under anesthesia ( $1 \%$ pentobarbital sodium was used for anesthesia at a dose of $50 \mathrm{mg} / \mathrm{kg}$, intraperitoneal injection), then, the tumor samples were collected for further analysis. All animal care and handling procedures were in accordance with the National Institutes of Health Guide for the Care and Use of Laboratory Animals (20) and were approved by the Ethics Committee of Hangzhou First People's Hospital.

TUNEL staining. TUNEL staining was used to detect apoptosis in the resected tissue samples. Frozen tumor tissue sections $\left(3-5 \mu \mathrm{m}\right.$; stored at $\left.-20^{\circ} \mathrm{C}\right)$ from the indicated nude mice were processed for TUNEL staining (Beyotime Institute of Biotechnology), according to the manufacturer's instructions. Briefly, following fixation by $4 \%$ paraformaldehyde for 20-30 min at room temperature, the frozen sections were washed three times with PBS at room temperature $(5 \mathrm{~min}$ each time). Then, sections were immersed in PBS containing $1 \%$ Triton X-100 for $20 \mathrm{~min}$ at room temperature. Each sample was incubated with $50 \mu \mathrm{l}$ TdT enzyme reaction solution (45 $\mu 1$ equilibration buffer, $1 \mu 1$ biotin-11-dUTP and $4 \mu 1$ TdT enzyme) for $60 \mathrm{~min}$ at RT. Following PBS washing, $50 \mu \mathrm{l}$ streptavidin-TRITC labeling buffer was added and incubated for $30 \mathrm{~min}$ at room temperature. Then, sections were covered with DAPI $(1: 1,000)$ for $10 \mathrm{~min}$ at RT. Finally, the sections were placed under a cover slip and scanned by Invitrogen EVOS FL AUTO (Thermo Fisher Scientific, Inc.).

Statistical analysis. The results are presented as the mean \pm SD and all experiments were repeated at least three times. Statistical analysis was performed using SPSS 16.0 (SPSS, Inc.). Differences between two groups were analyzed 
A

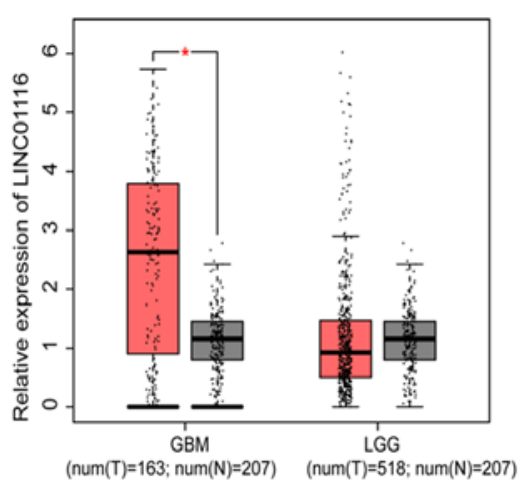

D

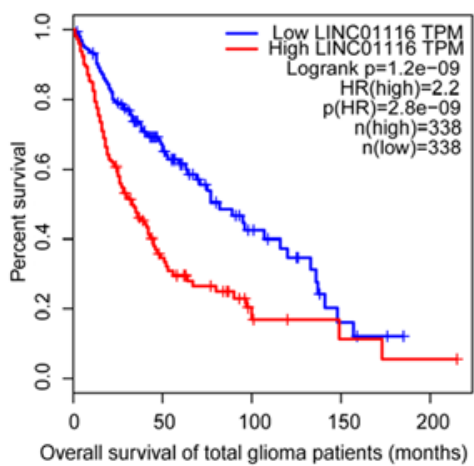

B

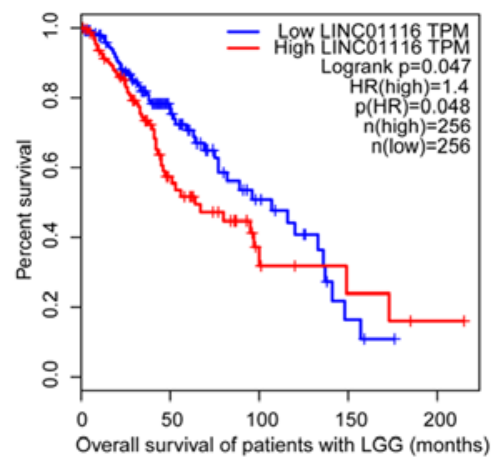

E

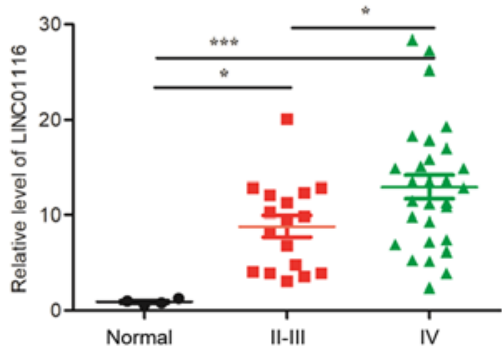

C

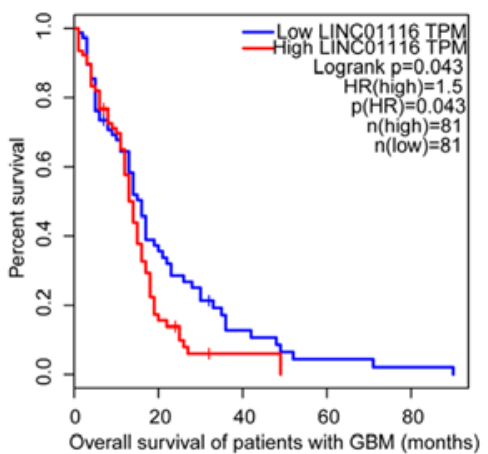

$\mathbf{F}$

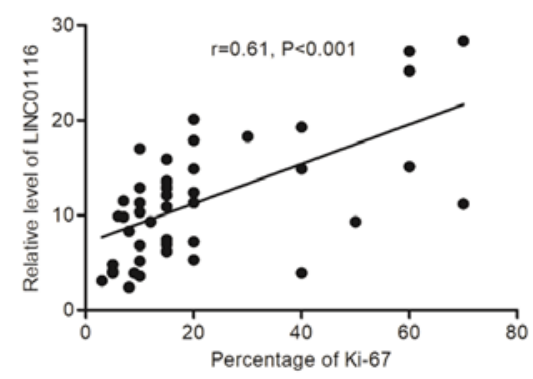

Figure 1. LINC01116 expression is upregulated in glioma and predicts poor prognosis. (A) Relative expression of LINC01116 in GBM and LGG was analyzed using the GEPIA platform. Compared with normal brain tissues, GBM tissues exhibited higher expression levels of LINC01116. (B-D) Overall survival analysis based on LINC01116 expression levels in different grade glioma tissues was also analyzed on the GEPIA platform. Regardless of glioma grade (i.e. total glioma patients), the survival times of patients with high LINC01116 were significantly shorter than those with low LINC01116 expression. (E) Relative expression of LINC01116 in 46 fresh glioma tissues. Both II-III grade gliomas $(\mathrm{n}=17)$ and GBMs $(\mathrm{n}=29)$ exhibited significantly higher LINC01116 expression levels than normal brain tissues $(\mathrm{n}=4)$. (F) Expression of LINC01116 was positively correlated with the percentage of Ki-67-positive cells in glioma tissues $(\mathrm{n}=46) .{ }^{*} \mathrm{P}<0.05,{ }^{* * *} \mathrm{P}<0.001$. LINC01116, long intergenic non-protein coding RNA 1116; GBM, glioblastoma; LGG, lower grade glioma; GEPIA, Gene Expression Profiling Interactive Analysis.

using a Student's t-test, and comparisons between two groups were analyzed by a oneway ANOVA, followed by Tukey's post hoc test. Correlations between two sets of data were analyzed using a Pearson's correlation analysis. A log-rank test was used for Kaplan-Meier curves. GraphPad Prism 5.0 software (GraphPad Software, Inc.) was used for data presentation. $\mathrm{P}<0.05$ was considered to indicate a statistically significant difference.

\section{Results}

LINC01116 is highly expressed in glioma. To determine the significance of LINC01116 in glioma, its expression and prognostic value were assessed in different grades of glioma [glioblastoma (GBM) and low-grade glioma (LGG)] using the GEPIA website. As shown in Fig. 1A, LINC01116 expression was significantly higher in GBM than in normal brain tissues. Although a similar result was not observed in LGG, there were still a number of these tissues with high levels of LINC01116 expression. In survival analysis, high levels of LINC01116 were found to predict poor prognosis, regardless of the glioma grade (Fig. 1B-D). To confirm whether LINC01116 is associated with a malignant glioma phenotype, its expression was assessed in 46 fresh glioma and 4 normal brain tissue samples. With a deviation from the results of GEPIA analysis, higher expression levels of LINC01116 were observed in low and high grade gliomas than in normal brain tissues (Fig. 1E), and LINC01116 expression was positively correlated with tumor grade and Ki-67 percentage positivity (Fig. 1F).

LINC01116 promotes the proliferation and invasion of glioma. To determine its function in glioma, RT-qPCR was used to assess the expression levels of LINC01116 in three glioma cell lines (U87, A172 and Shg44), compared with normal human astrocyte cells (HEB) (Fig. 2A). According to the expression of LINC01116 in glioma cells, a stable LINC01116 KD cell line was successfully constructed using lentiviral shRNA delivery into Shg44 cells with relative high expression of LINC01116, and stable OE cells were generated by introducing expression plasmids into A172 cells with relative low expression of LINC01116 (Fig. 2B and C). Functional experiments were then performed. Colony formation and cell viability assays revealed that the proliferative capacity of A172 cells was promoted by LINC01116 OE, and attenuated by LINC01116 KD in Shg44 cells (Fig. 2D-F). The results of the Transwell assay revealed that LINC01116 OE increased the invasive ability of A172 cells, while LINC01116 KD decreased that of Shg44 cells (Fig. 2F). These results indicated that LINC01116 may act as an oncogene in glioma.

LINC01116 inhibits the p53 pathway by upregulating MDM2. To investigate the potential tumor promoting mechanisms 
A

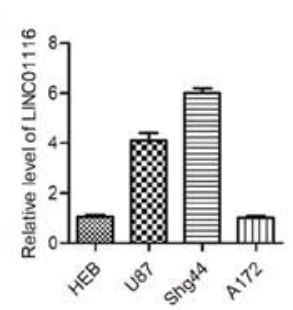

B

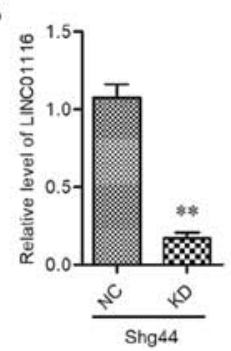

C

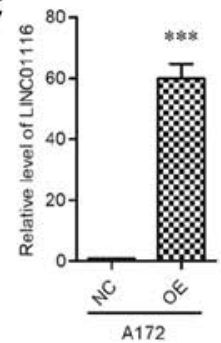

D

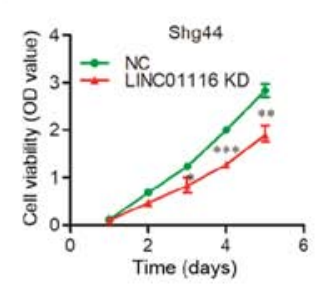

E

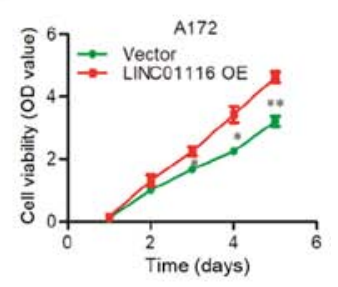

$\mathbf{F}$ NC LINC01116 KD Vector
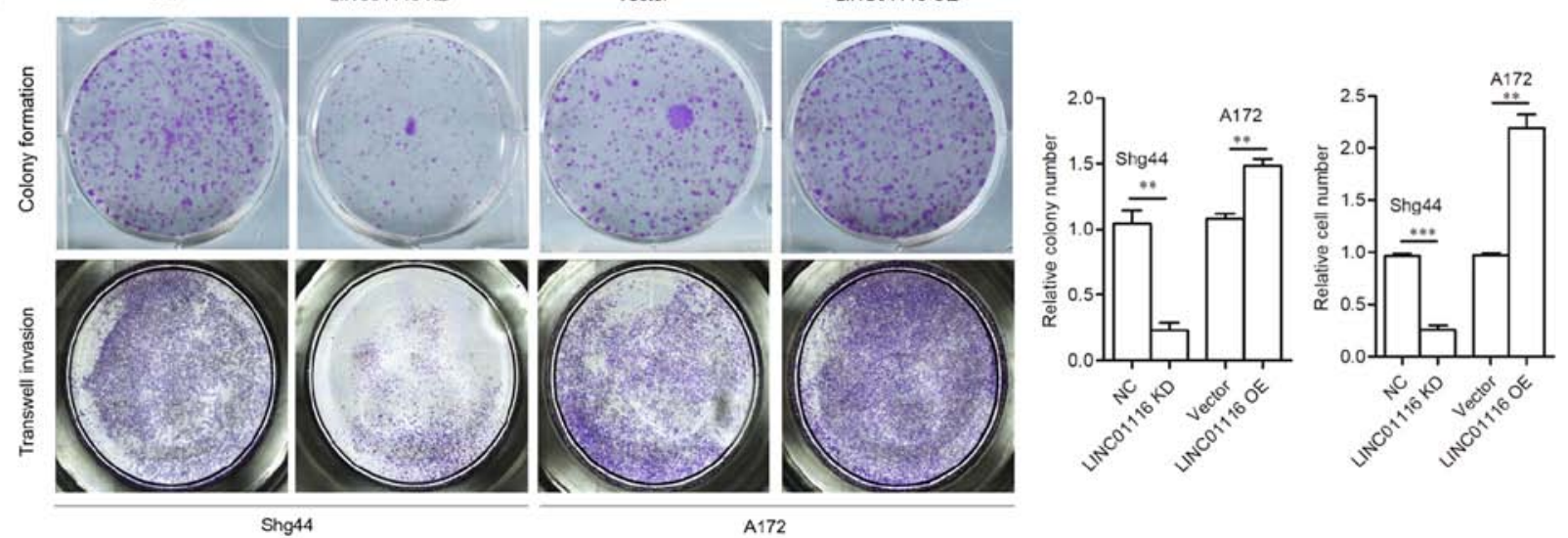

Figure 2. LINC01116 promotes the proliferation and invasiveness of glioma. (A) Relative expression of LINC01116 in HEB and glioma cell lines (U87, A172 and Shg44). Stable (B) LINC01116 KD and (C) LINC01116 OE cell lines were constructed using Shg44 and A172 cells, respectively. Relative LINC01116 expression in stable KD or OE cells was assessed by reverse transcription-quantitative PCR. Cell Counting Kit- 8 assays showed that (D) LINC01116 KD decreased Shg44 cell proliferation, whereas (E) LINC01116 OE promoted the proliferation of A172 cells. (F) Effects of LINC01116 KD and OE on the colony formation and invasive abilities of Shg 44 and A172 cells respectively. Histograms indicate the relative numbers of colonies and cells. ${ }^{*} \mathrm{P}<0.05,{ }^{* * *} \mathrm{P}<0.01$ and ${ }_{* * * *} \mathrm{P}<0.001$ vs. NC. LINC01116, long intergenic non-protein coding RNA 1116; KD, knockdown; OE, overexpression; NC, negative control.

of LINC01116, its association with the expression of proliferation- and invasion-related genes was investigated using the GEPIA platform. As shown in Fig. 3A, the mRNA expression levels of BAX, BAK1, CDKN1A and GADD45A were negatively associated with LINC01116 expression in glioma (Fig. 3A). Notably, these genes are all associated with the p53 pathway $(21,22)$. Thus, it was hypothesized that LINC01116 may promote the development of glioma by inhibiting the p53 pathway. To confirm these findings, the two p53-targeted-genes (BAK1 and GADD45A) with the most significant association with LINC01116 were chosen for further assays, combined with MDM2 and TP53. Then, the mRNA expression levels of BAK1, GADD45A, MDM2 and TP53 in LINC01116 OE cells (A172) and LINC01116 KD cells (Shg44) were determined. As shown in Fig. 3B and C, LINC01116 negatively regulated $B A K 1$ and $G A D D 45 A$, and positively regulated $M D M 2$ mRNA expression in glioma cells, but had no effect on TP53 mRNA levels. The protein expression levels of these genes were also determined, and those of BAK1, GADD45A and MDM2 were in accordance with their mRNA expression. However, p53 expression was decreased in LINC01116 OE cells and increased in LINC01116 KD cells (Fig. 3D and E). It was therefore speculated that LINC01116 may inhibit the p53 pathway by upregulating MDM2 in glioma. To verify this hypothesis, p53 protein expression was determined in LINC0111 OE cells following MDM2 KD. As shown in Fig. 3F and G, LINC01116 failed to repress the expression of p53, BAK1 and GADD45A protein in the absence of MDM2. These results indicated that
LINC01116 inhibited the p53 pathway in glioma by upregulating MDM2.

LINC01116 upregulates MDM2 by sponging miR-744-5p. One of the mechanisms by which IncRNAs exert their biological functions is by competitively sponging miRNAs, acting as ceRNAs. To investigate whether a ceRNA mechanism exists between LINC01116 and MDM2, the ENCORI platform (17) was used to identify miRNAs that potentially bound both LINC01116 and the 3'UTR of MDM2 mRNA. The miRNAs that could interact with LINC01116 and 3'UTR of MDM2 mRNA simultaneously were analyzed, which led to the identification of $\mathrm{miR}-744-5 \mathrm{p}$. To determine the association between LINC01116, miR-744-5p and MDM2, miR-744-5p expression was evaluated in A172 LINC01116 OE cells and Shg44 LINC01116 KD cells. LINC01116 was found to negatively regulate the expression of miR-744-5p in A172 and Shg44 cells (Fig. 4A). Next, Shg44 cells were transfected with miR-744-5p mimics and A172 cells were treated with a miR-744-5p inhibitor, RT-qPCR results showed that the relative miR-744-5p expression levels were significantly increased in Shg44 cells treated with miR-744-5p mimics and significantly reduced in A172 cells treated with miR-744-5p inhibitor (Fig. S1A and B). Then, the relative expression of LINC01116 and MDM2 mRNA was determined. As shown in Fig. 4B, miR-744-5p mimics significantly inhibited the expression of LINC01116 and MDM2 mRNA in Shg44 cells, whereas the miR-744-5p inhibitor promoted the expression of LINC01116 

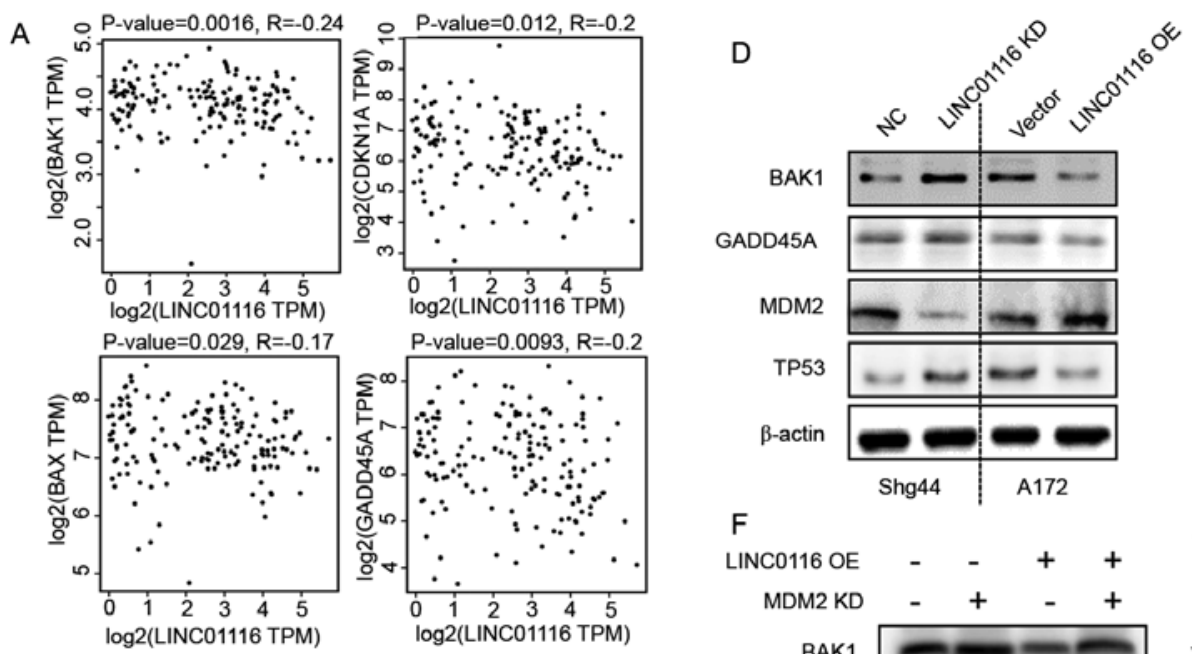

F

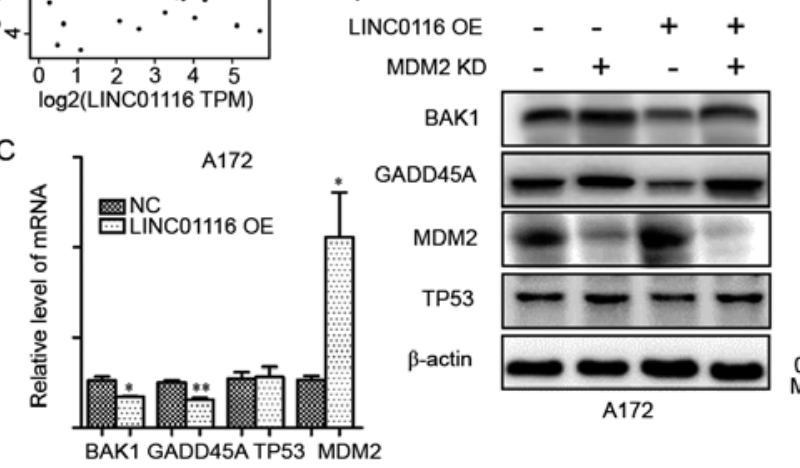

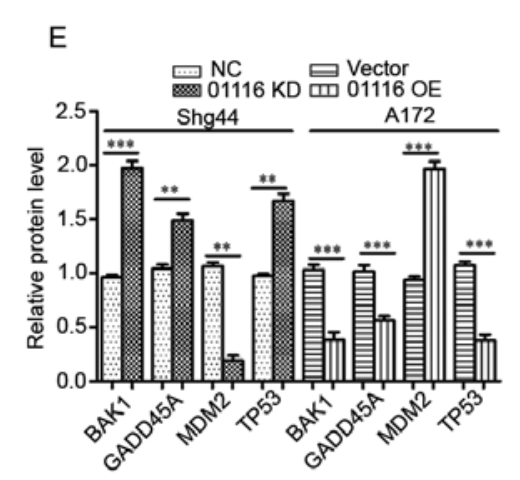

G

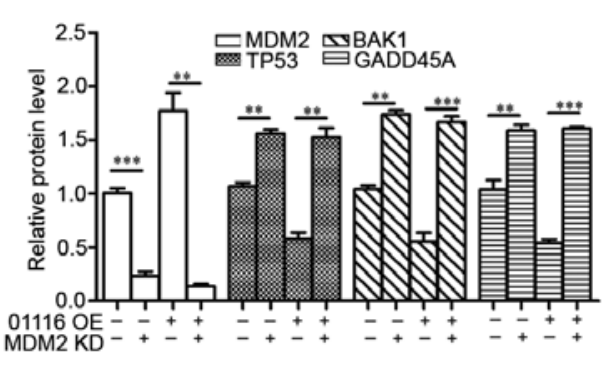

Figure 3. LINC01116 mediates the MDM2-p53 pathway in glioma. (A) Gene Expression Profiling Interactive Analysis revealed that LINC01116 levels negatively correlate with the mRNA levels of p53 target genes $B A K 1, B A X, C D K N 1 A$ and $G A D D 45 A$ in GBM tissues (n=81). (B and C) LINC01116 regulated the mRNA expression of $M D M 2, B A K 1$ and GADD45A, but not TP53 in glioma cell lines. (D) Protein expression of MDM2, p53, BAK1 and GADD45A were regulated by LINC01116 in glioma cells. (E) Histogram indicating the relative protein levels in (D). (F) LINC01116 regulated the p53 pathway in A172 cells, which was dependent on the presence of MDM2. (G) Histogram indicating the relative protein levels in $(\mathrm{F})$. ${ }^{*} \mathrm{P}<0.05$, ${ }^{* *} \mathrm{P}<0.01$ and ${ }^{* * * *} \mathrm{P}<0.001 \mathrm{vs} \quad \mathrm{NC}$. LINC01116, long intergenic non-protein coding RNA 01116; MDM2, E3 ubiquitin-protein ligase Mdm2; KD, knockdown; OE, overexpression; NC, negative control; CDKN1A, cyclin-dependent kinase inhibitor 1; GADD45A, growth arrest and DNA damage-inducible protein GADD45 $\alpha$.

and MDM2 mRNA in A172 cells. Furthermore, miR-744-5p mimics decreased the expression of MDM2 mRNA and protein promoted by LINC01116 OE in A172 cells, yet the miR-744-5p inhibitor increased the expression of MDM2 mRNA and protein downregulated by LINC01116 KD in the Shg44 cell line (Fig. 4C and D). A dual-luciferase reporter assay was then performed to determine whether miR-744-5p directly binds LINC01116 and the 3'UTR of MDM2. As shown in Fig. 4E, wild-type reporter plasmids were constructed containing the LINC01116 sequence and the 3'UTR of MDM2, as well as the corresponding mutant-type plasmids. The assay results showed that miR-744-5p mimics reduced the luciferase activity of the wild-type, but not the mutant-type plasmids (Fig. 4F and $\mathrm{G})$. In addition, LINC01116 OE reversed the attenuated luciferase activity of the MDM2 wild-type plasmid caused by the miR-744-5p mimics (Fig. 4F). However, miR-744-5p inhibitor did not significantly change the luciferase activity of LINC01116 wild or mutant-type plasmids (Fig. 4G). Finally, a RIP assay was performed to verify whether LINC01116 and miR-744-5p could bind to the same Ago protein. As shown in Fig. 4H, both LINC01116 and miR-744-5p were found to bind Ago2. These results indicated that LINC01116 upregulates MDM2 by sponging miR-744-5p.

miR-744-5p partially reverses the tumor-promoting ability of LINC01116 in glioma. After confirming the existence of a ceRNA mechanism between LINC01116 and miR-744-5p, the expression of miR-744-5p and its correlation with LINC01116 and Ki-67 in glioma were investigated. As shown in Fig. 5A, the expression of miR-744-5p was downregulated in glioma compared with normal brain tissues. Correlation analysis between miR-744-5p and Ki-67 showed that glioma tissues with low miR-744-5p expression displayed higher proliferative ability, although the correlation was weak ( $r=-0.347)$ (Fig. 5B). Next, the expression of miR-744-5p was detected in glioma cell lines (Fig. 5C); as a result, Shg44 cells were transfected with miR-744-5p mimics, and A172 cells were treated with an miR-744-5p inhibitor accordingly. As shown in Fig. 5D and E, cell proliferation was inhibited by miR-744-5p mimics and promoted by the inhibitor. Rescue experiments were performed to determine whether miR-744-5p could reverse the tumor-promoting role of LINC01116 in glioma. Firstly, the correlation between LINC01116 and miR-744-5p was analyzed, and the relative expression of miR-744-5p was found to be negatively correlated with that of LINC01116 (Fig. 5F). Rescue experiments revealed that miR-744-5p mimics could reverse the promotion of LINC01116 OE on the proliferative and invasive abilities of A172 cells (Fig. 5G and H). Similar results were obtained in Shg44 cells. Furthermore, findings also suggested that the LINC01116 KD-induced inhibition of proliferation and invasion was reversed by the miR-744-5p inhibitor (Fig. 5I and J).

LINC01116 promotes glioma development in vivo. After partially ascertaining the tumor-promoting mechanisms of 
A
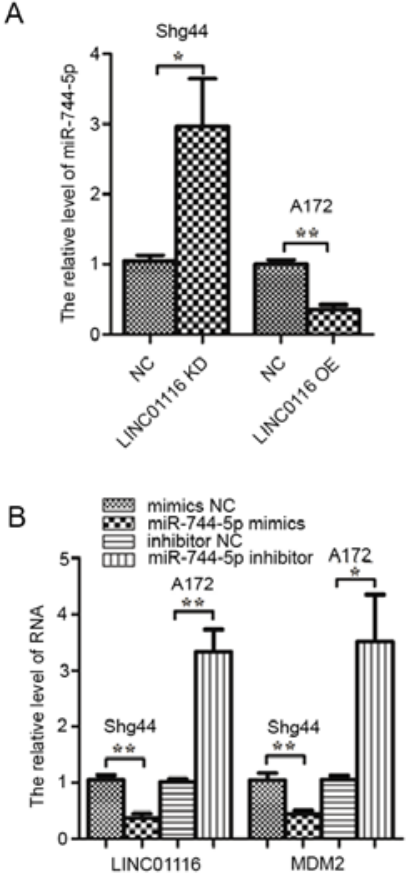
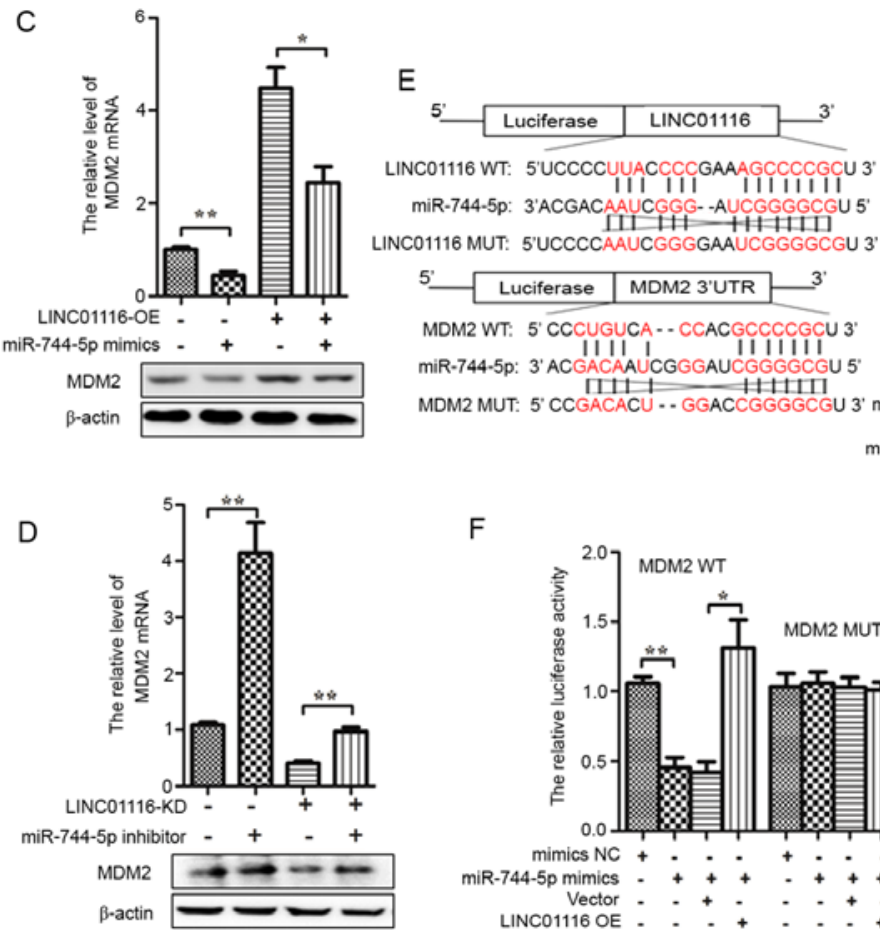

$\mathrm{F}$

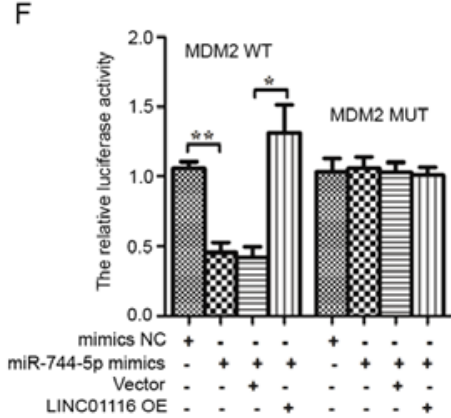

G

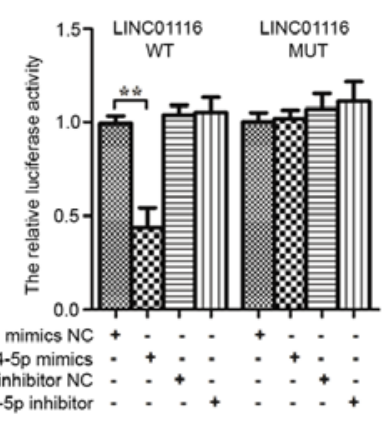

$\mathrm{H}$

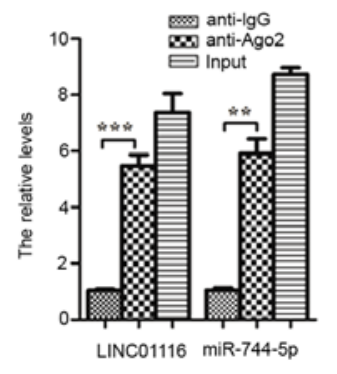

Figure 4. LINC01116 increases MDM2 mRNA levels by sponging miR-744-5p in glioma. (A) LINC01116 negatively regulated the expression of miR-744-5p in Shg44 and A172 cells. (B) mRNA expression of LINC01116 and MDM2 were downregulated by miR-744-5p mimics in Shg44 cells, and upregulated by the miR-744-5p inhibitor in A172 cells. (C) LINC01116 OE partially rescued the expression of MDM2 mRNA and protein downregulated by miR-744-5p mimics in A172 cells. (D) miR-744-5p inhibitors partially rescued MDM2 mRNA and protein expression downregulated by LINC01116 KD in Shg44 cells. (E) Predicted binding sites between miR-744-5p and LINC01116 or the MDM2 mRNA 3'-UTR in WT and MUT sequences. (F) miR-744-5p significantly reduced the luciferase activity of WT LINC01116 in 293T cells, but not the MUT sequence, and the reduction in luciferase activity was rescued by LINC01116 OE. (G) Luciferase activity of the WT MDM2 3'-UTR, but not the MUT, was significantly reduced by miR-744-5p mimics in 293T cells. However, miR-744-5p inhibitor did not significantly change the luciferase activity of LINC01116 WT or MUTplasmids. (H) RNA immunoprecipitation assays showed that both LINC01116 and miR-744-5p could bind Ago2. ${ }^{*} \mathrm{P}<0.05,{ }^{* *} \mathrm{P}<0.01$ and ${ }^{* * *} \mathrm{P}<0.001$. LINC01116, long intergenic non-protein coding RNA 01116; MDM2, E3 ubiquitin-protein ligase Mdm2; miR, microRNA; KD, knockdown; OE, overexpression; NC, negative control; UTR, untranslated region; WT, wild-type; MUT, mutant.

LINC01116 in glioma, its potential as a therapeutic target was investigated. A tumor xenograft model was established by subcutaneously injecting Shg44 LINC01116 KD cells into nude mice. As shown in Fig. 6A, LINC01116 KD impaired the growth of Shg44 cell tumors. To confirm whether LINC01116 exerted a similar effect on the expression of p53 pathway proteins, the mRNA and protein expression levels of these genes were evaluated in the tumors of nude mice. As predicted, the results were in accordance with those of the in vitro cellular experiments (Fig. 6B and C). In addition, TUNEL assay results revealed that LINC01116 KD increased the percentage of apoptotic cells in glioma tissues (Fig. 6D). Collectively, these findings suggested that targeting LINC01116 may be a novel approach for the treatment of glioma.

\section{Discussion}

The high mortality rate of glioma is primarily due to its infiltration and migration into a large area of adjacent brain tissue (23). Therefore, verifying the potential mechanisms involved in glioma development is necessary for future therapeutic advancements (2). To date, numerous studies have identified that IncRNAs possess pro- and/or antitumor functions in glioma (24-27), and identifying the regulatory effects of lncRNAs in glioma is a popular area of research.
Previous studies have demonstrated that LINC01116 is highly expressed in several types of cancer (28-30), and that it regulates cancer cell proliferation, migration and chemoresistance via a number of different mechanisms (28,29,31-37). In accordance with these studies, high LINC01116 expression was also observed in glioma in the present study, and was found to be positively associated with glioma grade and proliferative ability. Furthermore, LINC01116 KD inhibited the proliferative and invasive abilities of glioma cells, implying that LINC01116 is involved in the development and progression of glioma. Thus, the potential mechanisms through which LINC01116 is involved in gliomagenesis were investigated.

In a variety of cancer types, including glioma, the tumor suppressor p53 plays a crucial role in the development and progression of tumors, and its mutation or functional inactivation are found in the majority of human cancers (38). p53 acts as 'guardian of the genome' through surveillance and maintenance of genomic stability (39). As a key transcription factor, p53 triggers cell cycle arrest, senescence or apoptosis in response to DNA damage and various oncogenic stimuli (40). However, mutation of the TP53 gene or instability of the p53 protein contributes to tumor progression by producing dysfunctional $\mathrm{p} 53$ variants or accelerating p 53 degradation (41). Therefore, maintaining the stability and function of p53 is important for cellular homeostasis. In the present study, a 
A

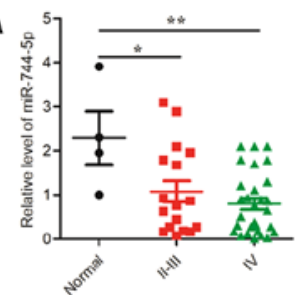

C

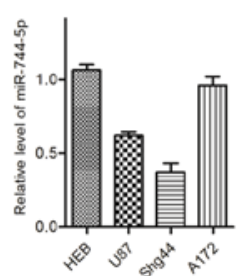

E

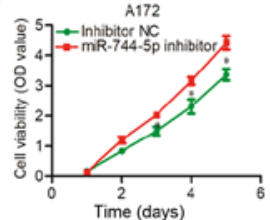

B

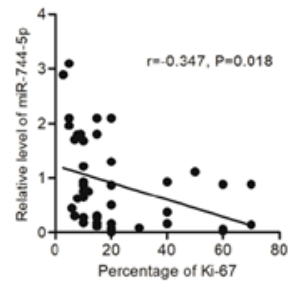

D

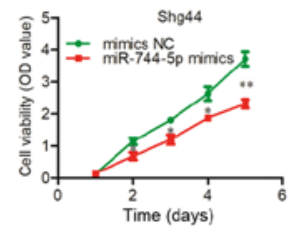

$\mathbf{F}$

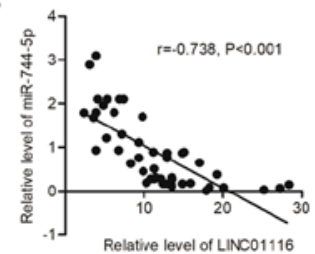

G

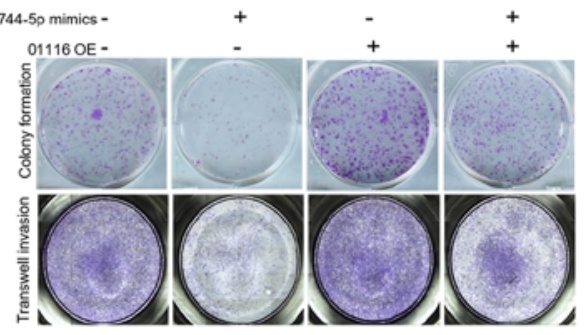

I 744-5p inhibitor -

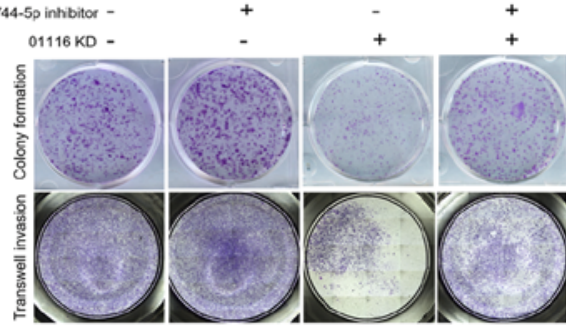

H

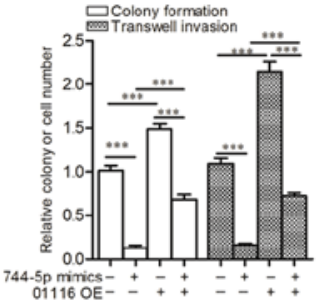

$\mathbf{J}$

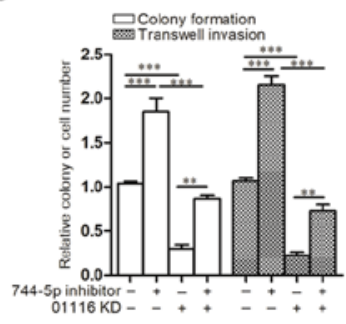

Figure 5. miR-744-5p inhibits the proliferation and invasiveness of glioma and reverses the biological functions of LINC01116. (A) miR-744-5p was significantly downregulated at different glioma grades. (B) miR-744-5p expression was negatively correlated with the percentage of Ki-67 in glioma tissues. (C) Relative levels of miR-744-5p in different glioma cell lines. Cellular proliferation was (D) reduced by miR-744-5p mimics in Shg44 cells, and (E) upregulated by the miR-744-5p inhibitor in A172 cells. (F) miR-744-5p expression was negatively correlated with LINC01116 expression in glioma tissues. (G) Colony formation and invasive ability of Shg44 cells were inhibited by miR-744-5p mimics, which was reversed by OE of LINC01116. (H) Histograms indicate the relative number of colonies and cells. (I) miR-744-5p inhibitor promoted the colony formation and invasive ability of A172 cells and partially restored these behaviors inhibited by LINC01116 KD. (J) Histograms indicate the relative number of colonies and cells. ${ }^{*} \mathrm{P}<0.05$, ${ }^{* *} \mathrm{P}<0.01$ and ${ }^{* * *} \mathrm{P}<0.001 \mathrm{vs} \mathrm{NC}$. miR, microRNA; LINC01116, long intergenic non-protein coding RNA 1116; KD, knockdown; OE, overexpression; NC, negative control.

A
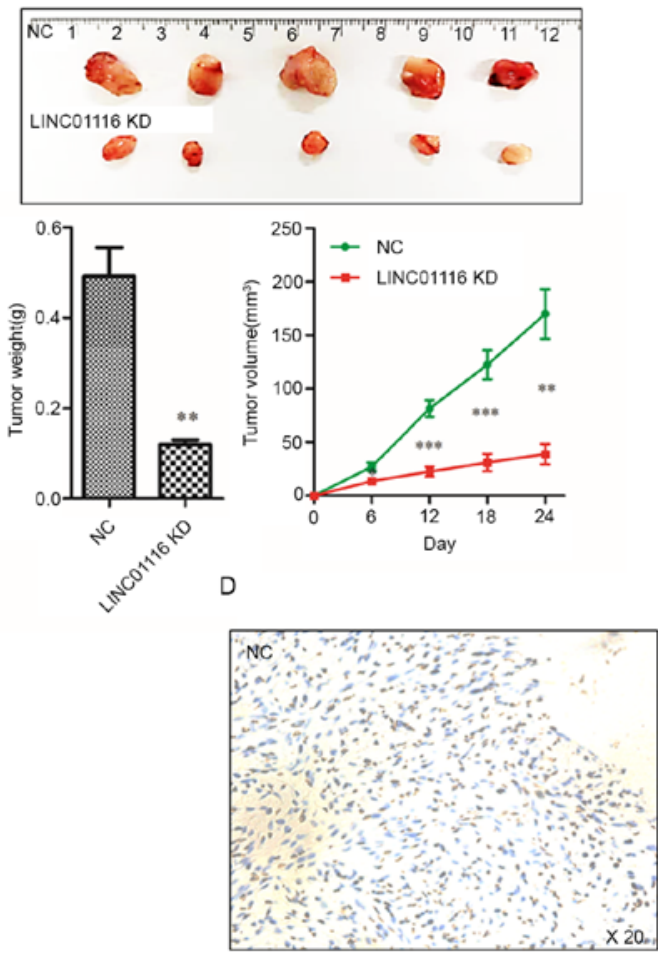
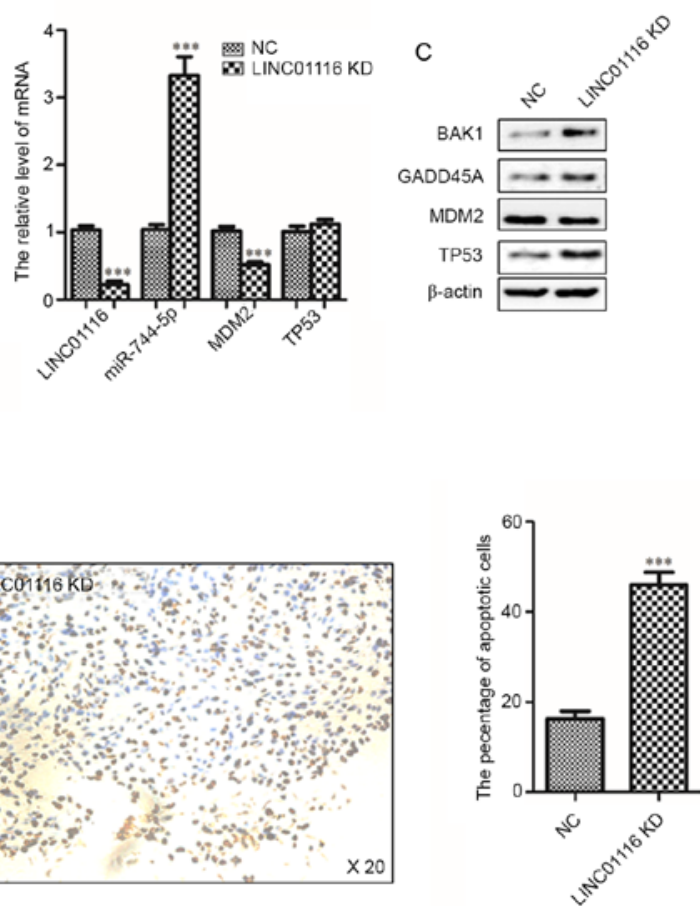

Figure 6. LINC01116 promotes gliomagenesis in vivo. (A) LINC01116 KD inhibited the growth of Shg44 cell tumors. Histogram indicating the mean weight of the xenograft tumors $(\mathrm{n}=5)$. Line chart of tumor growth curves $(\mathrm{n}=5)$. (B) mRNA expression levels of LINC01116, miR-744-5p, MDM2 and TP53 in the indicated xenograft tumors. (C) Protein expression of BAK1, GADD45A, MDM2 and p53 in xenograft tumors. (D) TUNEL assays were performed to detect glioma cell apoptosis in the xenograft tumors. Histogram indicating the percentage of apoptotic cells in the xenograft tumors ( $\mathrm{n}=5$ ). Magnification, x20. ${ }^{* *} \mathrm{P}<0.01$ and ${ }^{* * *} \mathrm{P}<0.001$ vs NC. LINC01116, long intergenic non-protein coding RNA 01116; miR, microRNA; KD, knockdown; NC, negative control; GADD45A, growth arrest and DNA damage-inducible protein GADD45 $\alpha$; MDM2, E3 ubiquitin-protein ligase Mdm2. 
negative association between the expression of LINC01116 and p53 target genes was identified, suggesting that LINC01116 may promote glioma proliferation and invasion by inhibiting the p53 pathway. LINC01116 was also confirmed to inhibit the expression of BAK 1 and GADD45A in three glioma cell lines. Notably, LINC01116 regulated the expression of $\mathrm{p} 53$ protein, but not p53 mRNA, which impelled the present study to focus on MDM2, an upstream regulator of the $\mathrm{p} 53$ pathway. As predicted, LINC01116 positively regulated the protein and mRNA expression of MDM2.

MDM2 functions as an E3 ubiquitin-ligase. It is highly expressed in various malignant tumors and is considered to be a proto-oncogene (42). MDM2 binds the transactivation domain of p53 and induces its degradation through the proteasomal system (43). Additionally, a number of proteins can repress the transcriptional activation of p53 by cooperating with MDM2 (44). In the present study, LINC01116 failed to regulate p53 without MDM2, which suggested that the regulatory role of LINC01116 in the p53 pathway was dependent on MDM2.

Various lncRNAs exert their biological functions via the ceRNA mechanism (45). To verify whether a ceRNA mechanism exists between LINC01116 and MDM2, miRNAs that potentially bind both LINC01116 and the 3'UTR of MDM2 were predicted using the StarBase platform. miR-744-5p was found to be a potential bridge between the regulation of LINC01116 and MDM2. Through further investigation, LINC01116 was found to regulate the mRNA expression of MDM2 by sponging miR-744-5p and promoting its degradation. miRNAs are small endogenous RNAs that control cellular and physiological processes by post-transcriptionally regulating gene expression (46). As miRNAs are crucial regulators of various tumorigenesis-associated genes, research into the functions of miRNA in tumors is expanding (47). Numerous miRNAs have been identified as promising candidate biomarkers for different types of cancer (48). In the present study, miRNA-744-5p was found to be downregulated in glioma tissue samples, and its expression was negatively associated with that of LINC01116. miR-744-5p also inhibited the proliferative and invasive abilities of glioma. Moreover, the pro-oncogenic functions of LINC01116 were attenuated by miR-744-5p OE. Those findings confirmed the presence of a ceRNA mechanism between LINC01116, miR-744-5p and MDM2. Given that targeting non-coding RNAs may be a potential approach to tumor treatment, LINC01116 KD was revealed to inhibit the growth of tumors in a nude mouse xenograft model. This finding suggested that LINC01116 may be a potential target for glioma treatment. Although miR-744-5p was found to link LINC01116 and the MDM2-p53 pathway, there were several limitations of the present study that need further study: i) The nature of the relationship between LINC01116 and p53 target genes (direct or undirect) was not determined; ii) only several p53-targeted genes were tested, and more genes related to the p53 pathway need to be explored in the future; iii) during the in vivo study, it was not tested whether miR-744-5p mimics could restore LINC01116-reduced tumor size; and iv) A172 cells with relatively low expression of LINC01116 were chosen for overexpression and Shg44 cells with relatively high expression were chosen for $\mathrm{KD}$, but it would have been more suitable to perform LINC01116 overexpression and KD in the same cell line.
In conclusion, these preliminary data demonstrated that LINC01116 was highly expressed in glioma, which was associated with a malignant phenotype. LINC01116 promoted the proliferation and invasiveness of glioma by sponging miR-744-5p, thus preserving MDM2 expression, which inhibited the antitumor functions of the p53 pathway. Therefore, targeting LINC01116 may be a potential future therapeutic approach for patients with glioma.

\section{Acknowledgements}

Not applicable.

\section{Funding}

No funding was received.

\section{Availability of data and materials}

The datasets used and/or analyzed during the current study are available from the corresponding author on reasonable request.

\section{Authors' contributions}

LJ, CC, WJ, HW, QD and XD performed the experiments. JS and WY designed the study and prepared the manuscript. JS and WY confirm the authenticity of all the raw data. All authors read and approved the final manuscript.

\section{Ethics approval and consent to participate}

The present study received approval from the Ethics Committee of Hangzhou First People's Hospital.

\section{Patient consent for publication}

Not applicable.

\section{Competing interests}

The authors declare that they have no competing interests.

\section{References}

1. Gusyatiner O and Hegi ME: Glioma epigenetics: From ubclassification to novel treatment options. Semin Cancer Biol 51: 50-58, 2018.

2. Aldape K, Brindle KM, Chesler L, Chopra R, Gajjar A, Gilbert MR, Gottardo N, Gutmann DH, Hargrave D, Holland EC, et al: Challenges to curing primary brain tumours. Nat Rev Clin Oncol 16: 509-520, 2019.

3. Lapointe S, Perry A and Butowski NA: Primary brain tumours in adults. Lancet 392: 432-446, 2018.

4. Post-transcriptional processing generates a diversity of 5'-modified long and short RNAs. Nature 457: 1028-1032, 2009.

5. Arun G, Diermeier SD and Spector DL: Therapeutic targeting of long non-coding RNAs in cancer. Trends Mol Med 24: 257-277, 2018.

6. Salmena L, Poliseno L, Tay Y, Kats L and Pandolfi PP: A ceRNA hypothesis: The rosetta stone of a hidden RNA language? Cell 146: 353-358, 2011

7. Qi X, Zhang DH, Wu N, Xiao JH, Wang X and Ma W: ceRNA in cancer: Possible functions and clinical implications. J Med Genet 52: 710-718, 2015. 
8. Abdollahzadeh R, Daraei A, Mansoori Y, Sepahvand M, Amoli MM and Tavakkoly-Bazzaz J: Competing endogenous RNA (ceRNA) cross talk and language in ceRNA regulatory networks: A new look at hallmarks of breast cancer. J Cell Physiol 234: 10080-10100, 2019.

9. Qu S, Liu Z, Yang X, Zhou J, Yu H, Zhang R and Li H: The emerging functions and roles of circular RNAs in cancer. Cancer Lett 414: 301-309, 2018.

10. Schmitt AM and Chang HY: Long noncoding RNAs in cancer pathways. Cancer Cell 29: 452-463, 2016.

11. Wang KC and Chang HY: Molecular mechanisms of long noncoding RNAs. Mol Cell 43: 904-914, 2011.

12. Peng $\mathrm{Z}$, Liu $\mathrm{C}$ and $\mathrm{Wu} \mathrm{M}$ : New insights into long noncoding RNAs and their roles in glioma. Mol Cancer 17: 61, 2018.

13. Lin $C$ and Yang L: Long Noncoding RNA in cancer: Wiring signaling circuitry. Trends Cell Biol 28: 287-301, 2018.

14. Kopp F and Mendell JT: Functional classification and experimental dissection of long noncoding RNAs. Cell 172: 393-407, 2018.

15. Tang Z, Li C, Kang B, Gao G and Zhang Z: GEPIA: A web server for cancer and normal gene expression profiling and interactive analyses. Nucleic Acids Res 45: W98-W102, 2017.

16. Livak KJ and Schmittgen TD: Analysis of relative gene expression data using real-time quantitative PCR and the 2(-Delta Delta C(T)) method. Methods 25: 402-408, 2001.

17. Li JH, Liu S, Zhou H, Qu LH and Yang JH: StarBase v2.0: Decoding miRNA-ceRNA, miRNA-ncRNA and protein-RNA interaction networks from large-scale CLIP-Seq data. Nucleic Acids Res 42: D92-D97, 2014

18. Peters L and Meister G: Argonaute proteins: Mediators of RNA silencing. Mol Cell 26: 611-623, 2007.

19. Filipowicz W, Bhattacharyya SN and Sonenberg N: Mechanisms of post-transcriptional regulation by microRNAs: Are the answers in sight? Nat Rev Genet 9: 102-114, 2008.

20. National Research Council: Guide for the Care and Use of Laboratory Animals. The National Academies Press, Washington, DC, 1996

21. Alavi MV: Targeted OMA1 therapies for cancer. Int J Cancer 145 2330-2341, 2019.

22. Thu HE, Hussain Z, Mohamed IN and Shuid AN: Eurycoma longifolia, a potential phytomedicine for the treatment of cancer: Evidence of p53-mediated apoptosis in cancerous cells. Curr Drug Targets 19: 1109-1126, 2018.

23. Jung E, Alfonso J, Osswald M, Monyer H, Wick W and Winkler F: Emerging intersections between neuroscience and glioma biology. Nat Neurosci 22: 1951-1960, 2019.

24. Rynkeviciene R, Simiene J, Strainiene E, Stankevicius V, Usinskiene J, Kaubriene EM, Meskinyte I, Cicenas J and Suziedelis K: Non-coding RNAs in glioma. Cancers (Basel) 11: $17,2018$.

25. Zheng Y, Xie J, Xu X, Yang X, Zhou Y, Yao Q and Xiong Y: IncRNA DDX11-AS1 exerts oncogenic roles in glioma through regulating miR-499b-5p/RWDD4 axis. Onco Targets Ther 14: 157-164, 2021

26. Wei Y, Zhou K, Wang C, Du X, Xiao Q and Chen C: Adsorption of miR-218 by lncRNA HOTAIR regulates PDE7A and affects glioma cell proliferation, invasion, and apoptosis. Int J Clin Exp Pathol 13: 2973-2983, 2020.

27. Wu YJ, Yang QS, Chen H, Wang JT, Wang WB and Zhou L: Long non-coding RNA CASC19 promotes glioma progression by modulating the miR-454-3p/RAB5A axis and is associated with unfavorable MRI features. Oncol Rep 45: 728-737, 2021

28. Wang H, Lu B, Ren S, Wu F, Wang X, Yan C and Wang Z: Long noncoding RNA LINC01116 contributes to gefitinib resistance in non-small cell lung cancer through regulating IFI44. Mol Ther Nucleic Acids 19: 218-227, 2020.

29. Hu HB, Chen Q and Ding SQ: IncRNA LINC01116 competes with miR-145 for the regulation of ESR1 expression in breast cancer. Eur Rev Med Pharmacol Sci 22: 1987-1993, 2018.
30. Chen J, Yuan ZH, Hou XH, Shi MH and Jiang R: LINC01116 promotes the proliferation and inhibits the apoptosis of gastric cancer cells. Eur Rev Med Pharmacol Sci 24: 1807-1814, 2020.

31. Xing H, Sun H and Du W: LINC01116 accelerates nasopharyngeal carcinoma progression based on its enhancement on MYC transcription activity. Cancer Med 9: 267-277, 2020

32. Zhang ZF, Xu HH, Hu WH, Hu TY and Wang XB: LINC01116 promotes proliferation, invasion and migration of osteosarcoma cells by silencing p53 and EZH2. Eur Rev Med Pharmacol Sci 23: 6813-6823, 2019.

33. Wu J, Chen Z, Zhang L, Cao J, Li X, Gong Z, Bo H, Zhang S and He D: Knockdown of LINC01116 inhibits cell migration and invasion in head and neck squamous cell carcinoma through epithelial-mesenchymal transition pathway. J Cell Biochem 121: $867-875,2020$

34. Chen Z, Tao Q, Qiao B and Zhang L: Silencing of LINC01116 suppresses the development of oral squamous cell carcinoma by up-regulating microRNA-136 to inhibit FN1. Cancer Manag Res 11: 6043-6059, 2019.

35. Ye J, Zhu J, Chen H, Qian J, Zhang L, Wan Z, Chen F, Sun S, Li $W$ and Luo C: A novel lncRNA-LINC01116 regulates tumorigenesis of glioma by targeting VEGFA. Int J Cancer 146: 248-261, 2020.

36. Fang YN, Huang ZL, Li H, Tan WB, Zhang QG, Wang L and Wu JL: LINC01116 promotes the progression of epithelial ovarian cancer via regulating cell apoptosis. Eur Rev Med Pharmacol Sci 22: 5127-5133, 2018.

37. Zhang B, Yu L, Han N, Hu Z, Wang S, Ding L and Jiang J: LINC01116 targets miR-520a-3p and affects IL6R to promote the proliferation and migration of osteosarcoma cells through the Jak-stat signaling pathway. Biomed Pharmacother 107: 270-282, 2018.

38. Wade M, Li YC and Wahl GM: MDM2, MDMX and p53 in oncogenesis and cancer therapy. Nat Rev Cancer 13: 83-96, 2013.

39. Tiwari B, Jones AE and Abrams JM: Transposons, p53 and genome security. Trends Genet 34: 846-855, 2018.

40. Hafner A, Bulyk ML, Jambhekar A and Lahav G: The multiple mechanisms that regulate p53 activity and cell fate. Nat Rev Mol Cell Biol 20: 199-210, 2019

41. Stiewe T and Haran TE: How mutations shape p53 interactions with the genome to promote tumorigenesis and drug resistance. Drug Resist Updat 38: 27-43, 2018.

42. Oliner JD, Saiki AY and Caenepeel S: The role of MDM2 amplification and overexpression in tumorigenesis. Cold Spring Harb Perspect Med 6: a026336, 2016.

43. Wu X, Bayle JH, Olson D and Levine AJ: The p53-mdm-2 autoregulatory feedback loop. Genes Dev 7: 1126-1132, 1993.

44. Hernandez-Monge J, Rousset-Roman AB, Medina-Medina I and Olivares-Illana V: Dual function of MDM2 and MDMX toward the tumor suppressors p53 and RB. Genes Cancer 7: 278-287, 2016.

45. Zhao J, Li L, Han ZY, Wang ZX and Qin LX: Long noncoding RNAs, emerging and versatile regulators of tumor-induced angiogenesis. Am J Cancer Res 9: 1367-1381, 2019.

46. Adams BD, Parsons C, Walker L, Zhang WC and Slack FJ: Targeting noncoding RNAs in disease. J Clin Invest 127: 761-771, 2017.

47. Bracken CP, Scott HS and Goodall GJ: A network-biology perspective of microRNA function and dysfunction in cancer. Nat Rev Genet 17: 719-732, 2016.

48. Anfossi S, Babayan A, Pantel K and Calin GA: Clinical utility of circulating non-coding RNAs - an update. Nat Rev Clin Oncol 15: 541-563, 2018.

This work is licensed under a Creative Commons Attribution-NonCommercial-NoDerivatives 4.0 International (CC BY-NC-ND 4.0) License. 